\title{
Using nonlinear features for fetal heart rate classification
}

\author{
J. Spilka ${ }^{\mathrm{a}, *}$, V. Chudáček ${ }^{\mathrm{a}}$, M. Kouckýb ${ }^{\mathrm{b}}$ L. Lhotskáa ${ }^{\mathrm{a}}$ M. Huptych ${ }^{\mathrm{a}}$, P. Jankůc, G. Georgoulas ${ }^{\mathrm{d}}$, C. Stylios ${ }^{\mathrm{d}}$ \\ ${ }^{a}$ Department of Cybernetics, Czech Technical University in Prague, Technicka 2, Prague 6, 16627 \\ ${ }^{b}$ Obstetrics and Gynaecology clinic, $1^{\text {st }}$ Medical Faculty of Charles University in Prague, Czech Republic \\ ${ }^{c}$ Obstetrics and Gynaecology clinic, University Hospital in Brno, Czech Republic \\ ${ }^{d}$ Department of Informatics and Communications Technology, Technological Educational Institution of Epirus, Artas, Greece
}

\begin{abstract}
Fetal heart rate (FHR) is used to evaluate fetal well-being and enables clinicians to detect ongoing hypoxia during delivery. Routine clinical evaluation of intrapartum FHR is based on macroscopic morphological features visible to the naked eye. In this paper we evaluated conventional features and compared them to the nonlinear ones in the task of intrapartum FHR classification. The experiments were performed using a database of 217 FHR records with objective annotations, i.e. pH measurement. We have proven that the addition of nonlinear features improves accuracy of classification. The best classification results were achieved using a combination of conventional and nonlinear features with sensitivity of $73.4 \%$, specificity of $76.3 \%$, and F-measure of 71.9\%. The best selected nonlinear features were: Lempel Ziv complexity, Sample entropy, and fractal dimension estimated by Higuchi method. Since the results of automatic signal evaluation are easily reproducible, the process of FHR evaluation can become more objective and may enable clinicians to focus on additional non-cardiotocography parameters influencing the fetus during delivery.
\end{abstract}

Keywords:

fetal heart rate, cardiotocography, nonlinear methods, feature selection, classification.

\section{Introduction}

Accurate evaluation of fetal status, based on available information, is crucial when difficulties occur during delivery. Even though a fetus is equipped with a defense mechanism to tackle the delivery induced stress, in some cases only timely intervention can prevent potential long-term consequences such as cerebral palsy, neuro-development disability, neonatal encephalopathy or even death, resulting from excessively long oxygen insufficiency [1, 2].

The introduction of cardiotocography (CTG - recording of fetal heart rate (FHR) and force/pressure of contractions) in the 1960's was accompanied by great expectation since it offered a new continuous fetal surveillance method. However, metaanalysis of large multi-centric studies [3] did not prove any significant improvements in the delivery outcomes. Some studies even disproved any evidence of advantages of continuous monitoring compared to intermittent one. Moreover, CTG became the main suspect for an increased rate of cesarean sections [4]. Therefore, in 1986 CTG interpretation guidelines were introduced in order to lower the number of asphyxiated neonates as well as the number of cesarean sections [5, 6]. Nowadays CTG remains the most prevalent method for intrapartum fetal surveillance [7, 8], often supported by ST-analysis method (Neoventa Medical, Sweden) which is based on analysis of fetal

\footnotetext{
${ }^{*}$ Corresponding author, Tel.: +420 224357325

Email address: spilka.jiri@fel.cvut.cz (J. Spilka)
}

electrocardiogram (fECG). The introduction of additional STanalysis into the clinical practice improved the labor outcomes slightly $[9,10]$ but its use is not always possible or feasible since it requires invasive measurement.

A recently published paper [4] concluded that the weakness of CTG still lies in a generally poor standard of interpretation and the contribution of the human factor, demonstrated by high intra and inter observer variability. Either more education and training on CTG interpretation should be performed [1, 2] or one should use a more cost-effective solution by developing a decision support system serving as a source of additional information [4].

First attempts for automatic analysis were completely based on clinical guidelines for CTG assessment [5]. Recently, other methods such as those derived from adults HRV research were used for FHR analysis [11]. The statistical description of CTG tracings was employed in [12] and in [11]. A short overview of papers which analyzed the spectrum of FHR either antepartum or intrapartum was published in [13]. An extension of frequency analysis, the wavelet transform, was employed in $[14,15]$. Recently, a system identification approach to estimate parameters from FHR and uterine pressures was described in [16].

Use of nonlinear methods for FHR analysis has its roots in adults HRV research. The measure of fractal dimension was performed by $[17,18,19]$. Another attempt was to measure the length of FHR curve using the Higuchi method [20]; the different estimations of fractal dimension were reviewed 
by [21]. Probably the most successful nonlinear methods for FHR analysis are approximate entropy (ApEn) and sample entropy (SampEn). They are widely used for examination of nonlinear systems and also proved their applicability in FHR analysis $[22,23,24]$. Another method for nonlinear analysis is Lempel Ziv complexity used by [25].

For classification of the FHR many different approaches were used, e.g. Support Vector Machines [15, 16, 26], hybrid approach utilizing grammatical evolution [27], and also conventional methods, such as k-nn (k-nearest neighbors), ldc (linear discriminant classifier), and qde (quadratic discriminant classifier) [26].

There are also several complete systems for fetal assessment. Among the best known are: Omniview SisPorto $®$ developed at University of Porto, Portugal [28] and the NST-Expert (NonStress Test) and its successor CAFE (Computer Aided Fetal Evaluation) [29]. Both systems use a holistic approach to CTG evaluation including information about the state of the mother. Processing of the FHR in these systems is mainly based on morphological features provided by FIGO guidelines.

The purpose of our work is to distinguish between two groups: normal fetuses and fetuses with developed acidemia. The general idea of this paper is not novel; most of the features were used in papers before (cited in previous paragraph) though they were usually employed in ad-hoc settings and tested on small datasets. In this paper we provide a general approach using a sufficiently large dataset and we evaluate a comprehensive set of features that originated from different domains. In addition, we consider thoroughly the applicability of nonlinear methods to FHR.

\section{Data description}

Data was acquired at the Dept. of Obstetrics and Gynaecology of the General University Hospital in Prague during 2007 and 2009; all women signed informed consent. The FHR signals used in this work were measured using Neoventa's STAN S21 system.

Recordings were checked for patient anamnesis and only one fold pregnancies delivered during the 38 th -42 nd week of pregnancy were chosen for the final database. Umbilical artery $\mathrm{pH}$ values were obtained to serve as objective evaluation of hypoxia. The neonatal acidemia is defined as $\mathrm{pH}$ below $7.05-$ these values were suggested in the work of Sundstrom [30]. Nevertheless there exist other works suggesting different values 7.10 [31], 7.15 [32]. Considering these facts and after consultation with obstetricians at the General University Hospital value of $\mathrm{pH}$ lower than 7.15 was considered not normal - further referred to as pathological.

Our database contained 217 recordings. Arterial $\mathrm{pH}$ values were available for all records and, based on $\mathrm{pH}$ threshold, 94 were considered as pathological. For comparison the expert annotations were gathered, using an in-house developed annotation system, from three experts in the field of obstetrics in the Czech Republic. Two measures were used for evaluation: intra-observer agreement as a percentage of consistently annotated records to all annotations and inter-observer agreement as a percentage of equally annotated records among the three experts to all annotations.

\section{Signal preprocessing}

Signals were measured either externally using Doppler ultrasound or internally by scalp electrode. Fetal heart rate recorded externally has lower signal to noise ratio than that recorded internally but there is no clinical difference between these two approaches.

Values of extracted features and further classification are highly dependent on the quality of signal preprocessing. Preprocessing steps could distort the data and add stochastic components making the use of nonlinear methods unsuitable.

In our case, the preprocessing consisted of three main steps: segment selection, artefacts removal and interpolation. An appropriate segment should be chosen as close as possible to delivery because during the last minutes major changes in fetal condition can occur. However, as it is shown in Figure 1, FHR directly preceeding the delivery is largely contaminated with artefacts and noise. Therefore, we evaluated the signal in terms of quality and automatically chose a segment with sufficient quality that is closest to delivery. Segments were 20 minutes long, i.e. 4800 samples using $4 \mathrm{~Hz}$ sampling frequency.
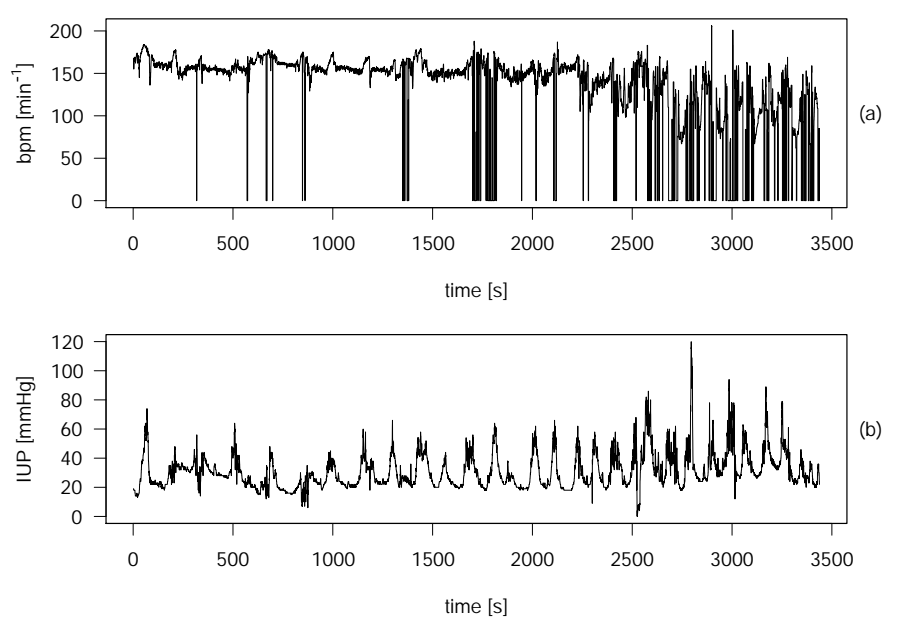

Figure 1: Raw record of CTG. (a) Fetal heart rate, (b) intrauterine pressure.

The FHR signal contains a lot of artefacts caused by mother and fetal movements or displacements of the transducer. In general, the amount of data being removed as artefacts or missing values is in the range between $20 \%-40 \%$ of all data. The algorithm suggested by Bernades et al. [33] was used for artefact removal. Any successive five beats with a difference lower than $10 \mathrm{bpm}$ among them are considered as a stable segment. Then, whenever the difference between adjacent beats is higher than $25 \mathrm{bpm}$, the sample is substituted by linear interpolation between the previous beat and the new stable segment. Thus, all abrupt changes in FHR are removed and replaced. The result of artefacts removal is presented in Figure 2. 
We used cubic Hermite spline interpolation [34], implemented in MATLABR, to replace the missing data. We did not compute across a gap [35] when the length of the missing data was 20 seconds or more - the value obtained based on our experiments. For the purpose of this paper cubic Hermite spline interpolation was used for equidistant $4 \mathrm{~Hz}$ data re-sampling since it is convenient for signal analysis and also it is the least prone to errors when frequency spectrum is estimated [36]. The spline interpolation also introduces nonlinearity, however, the amount of nonlinearity should be approximately the same for normal and pathological FHR.
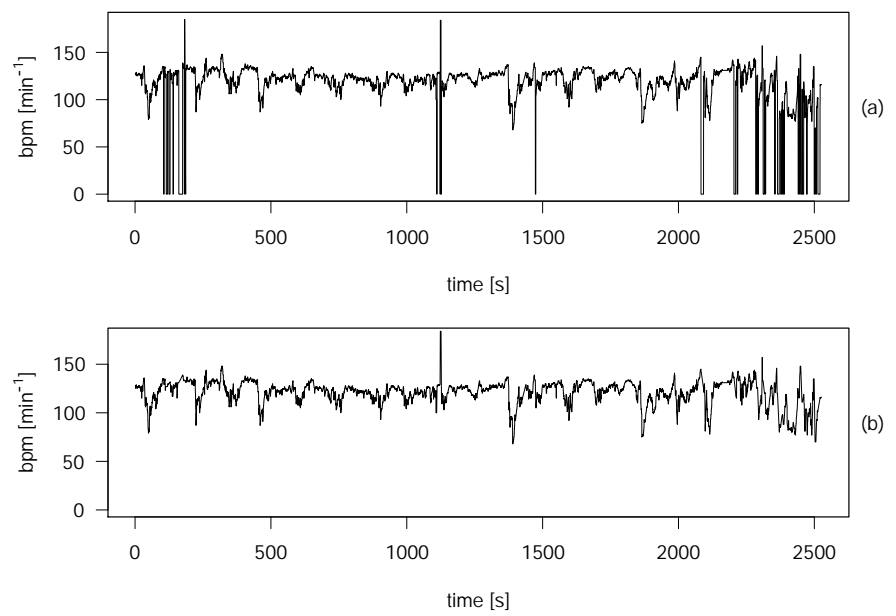

Figure 2: Removing of artefacts. (a) Raw signal with artefacts, (b) signal after artefacts removal.

For the use of nonlinear methods signals were detrended using second order polynomial, estimated, such that uninteresting trend was removed but interesting dynamics preserved. Also we normalized signals to zero mean and unit variance.

Despite the stress caused by delivery, the FHR is also affected by fetal activity (active and quiet state) as described by [37]. Nevertheless, the fetus is mostly awake during delivery, therefore, we neglected changes in FHR caused by activequiet states. We also neglected sex differences of FHR since we expected the difference between normal and pathological fetuses to be more profound [38].

\section{Conventional features}

To be able to compare our results to those of clinical praxis, we computed the morphological features introduced by the FIGO guidelines [5]. These features describe shape and changes of the FHR baseline such as baseline mean and median, number of accelerations and decelerations, long-term and short term variability, interval index, etc. Detailed description of these features is not the purpose of this paper and can be found in $[11,32]$.

Another type of features were those used routinely in adult HRV evaluation such as NN50, RMSSD, and Poincaré plot as well as the frequency features describing the amounts of energy in different energy bins. The power spectral density was estimated using the fast Fourier transform (FFT). For more information about statistical HRV features refer to [39] and about frequency bands to [37]. Note that Poincaré plot is a nonlinear feature that is commonly used in HRV; hence it is considered as conventional. The nonlinear features were computed over the whole 20 minutes segments of the preprocessed data.

\section{Nonlinear features}

The nonlinear approach may reveal relevant clinical information of FHR hidden to conventional time series analysis. Goldberger et al. [40] observed that a human heart beat fluctuates on different time scales and is self-similar; despite that there remains ongoing controversy over whether a normal heart rate is chaotic or not [41], tools used for examination of chaotic time series could also be useful for FHR analysis. There exist several approaches for nonlinear time series analysis; in this work fractal dimension, entropy, and complexity measures were utilized. When analyzing FHR by nonlinear methods we have to be aware of at least two major pitfalls. First, FHR contains stochastic components induced by motion artefacts and measurement process especially when an external ultrasound electrode is used for signal acquisition. These distortions could severely damage the nature of FHR; therefore we used a surrogate data test to establish nonlinearity of FHR. Second, a certain data length is necessary to reliably estimate values of nonlinear methods. The required data length for each method is discussed in the corresponding sections below.

\subsection{Fractal dimension}

There are two approaches to estimate dimension of a signal either by direct measurement of the waveform or by operating in reconstructed state space. The former approach considers a signal in $\mathbb{R}^{2}$ as a geometric object and directly uses it without any further transformation. On the other hand the state space is reconstructed from coordinates representing the variables needed to specify the state of a dynamical system.

The state space can be reconstructed using Taken's embedding theorem [42]. It states that it is possible to reconstruct state space from signal $x(t)$ delayed by time $\tau$ as long as the embedding dimension $m$ is larger than $2 d+1$, where $d$ is a box counting dimension. $x(t) \rightarrow y(t)=[x(t), x(t+\tau), \ldots, x(t+(m-1) \cdot \tau)]$. Different choice of $\tau$ and $m$ leads to different reconstruction. We adopted a mutual information approach [43] to search $\tau$ and considered a first marked minimum as the optimal time delay. For examination of embedding dimension we can use the Cao's method [44]. However, the correlation dimension, $D_{2}$, is invariant to $m$. It is therefore convenient to estimate $D_{2}$ for different $m$, e.g. increasing from 2 to 16 , and observe when a value of $D_{2}$ saturates. This approach is computationally less efficient but minimizes errors introduced by a single $m$ estimate. The state space reconstruction from FHR for normal and pathological fetus is shown in Figure 3; see figure caption for details.

There are many theories about required data length with a general agreement that required data length increases exponentially with data dimension. We followed data size requirements 

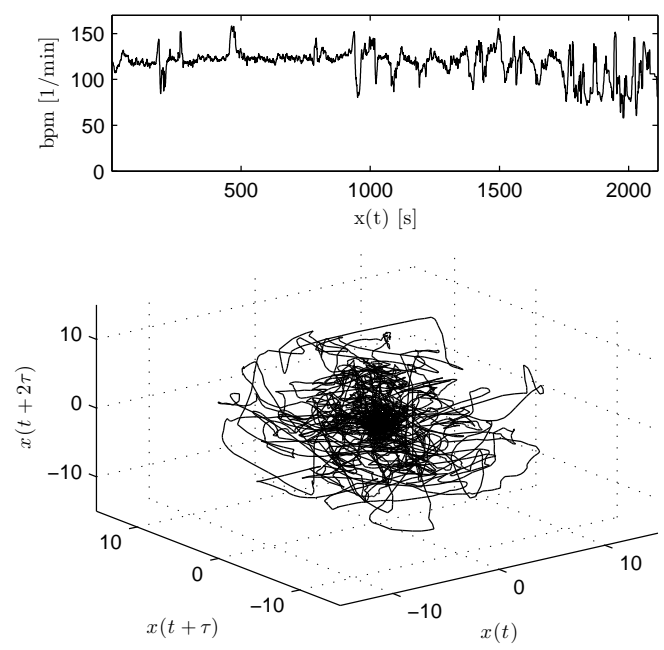

(a)
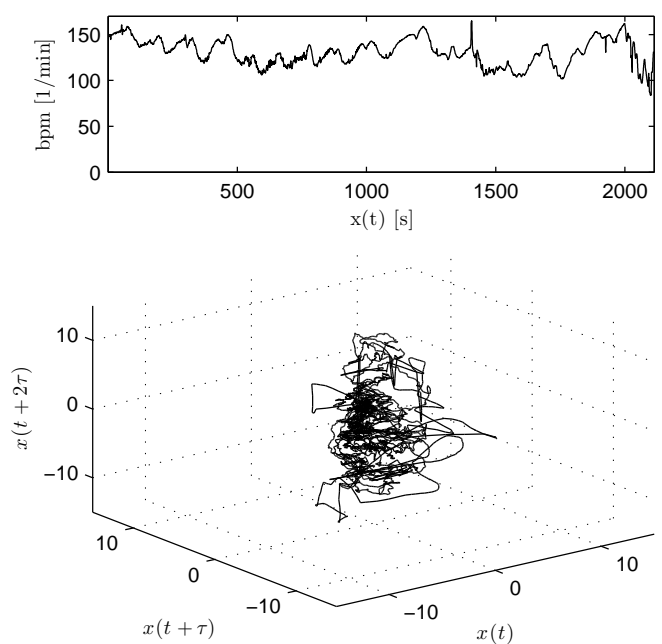

(b)

Figure 3: Fetal heart rate and state space reconstruction for normal and pathological fetus. The upper left and right signals represent fetal heart rate. The corresponding state space is shown below. (a) FHR and state space for normal fetus. The optimal delay time was $\tau=2.5 s$. (b) FHR and state space for pathological fetus. The optimal delay time was $\tau=5 \mathrm{~s}$. The low complexity of FHR for fetus with developed acidemia, (b), is clearly visible in both time and state space. In the state space the delayed coordinates of FHR span less area thus showing reduced variability.

suggested by [45]; for estimating a dimension $d$, a minimum data length $N_{\min }=10^{d / 2}$ is required.

\subsubsection{Correlation dimension}

Correlation dimension $D_{2}$ is based on an estimation of the correlation sum $C(r)$ which gives the probability that two randomly chosen points are close to each other with a distance smaller than $r$.

$$
C(m, r)=\frac{1}{N(N-1)} \sum_{i}^{N} \sum_{i>j}^{N} \Theta\left(r-\left\|\mathbf{y}_{\mathbf{i}}-\mathbf{y}_{\mathbf{j}}\right\|\right),
$$

where $\mathbf{y}$ are $m$-dimensional delay vectors, $N$ the number of points, and $\Theta$ is the Heaviside function: $\Theta(x)$ equals zero for $x<0$ and one for $x \geq 0$. If we consider the limit when $r$ approaches zero (theoretically) the correlation dimension is estimated as: $D_{2}=\lim _{r \rightarrow 0} \frac{\log C(r)}{\log r}$. The $D_{2}$ is usually estimated as a slope on $\log -\log$ plot where $\log C(r)$ is plotted as a function of $\log r$. Since the $D_{2}$ cannot be estimated for all radii we used local slope approach to estimate a proper scaling region $\left(r_{l}, r_{u}\right)$. Also we employed Takens-Theiler estimator [46, 47] which uses the maximum likelihood approach:

$$
D_{2}=\frac{C\left(r_{u}\right)-C\left(r_{l}\right)}{\int_{r_{l}}^{r_{u}} \frac{C(r)}{r} d r}
$$

\subsubsection{Box-counting dimension}

The box-counting dimension expresses the relationship between the number of boxes that contain part of an object and the size of the boxes. In the case of a signal, the minimal number of boxes $N$ of side length $\epsilon$, needed to cover whole signal is counted and then the side length of the boxes is decreased. The box counting dimension is estimated as follows: $D_{0}=\lim _{\epsilon \rightarrow 0} \frac{\log N}{\log (1 / \epsilon)}$, though $\epsilon$ could not reach zero for real signal.

\subsubsection{Higuchi's dimension}

The Higuchi method [48] calculates fractal dimension from the estimated length of a signal. From an original signal $x(1), x(2), \ldots, x(N)$ of length $N$ a new signal, $X_{k}^{m}$, is constructed: $X(m), X(m+k), X(m+2 k), \ldots, X(m+$ $[(N-m) / k]) \quad(m=1,2, \ldots, k)$, where [] denotes the Gauss' notation, $m$ defines the initial time, and $k$ the time interval. The $k$ represents time displacement and the number of new created subsets is equal to $k$. Then for each $m$ the length $L_{m}(k)$ of $X_{k}^{m}$ is computed. The length of the curve for time interval $k,\langle L(k)\rangle$, is defined as the average value over $k$ set of $L_{m}(k)$ :

$$
\langle L(k)\rangle=\sum_{m=1}^{k} L_{m}(k) / k
$$

The computed curve length $\langle L(k)\rangle$ for different $k$ is related to the fractal dimension $D$ by the exponential formula $\langle L(k)\rangle \propto$ $k^{-D}$. The fractal dimension is estimated as a slope of fitted regression to log-log plot of $\langle L(k)\rangle$ versus $k$.

\subsubsection{Variance dimension}

The variance fractal dimension is based on properties of fractional Brownian motion (fBm). Let the signal $x(t)$ be continuous in the time $t$ and $\Delta t$ is the time increment. The variance $\sigma^{2}$ is related to the time increments $\Delta t$ of signal $x(t)$ according to the power law [49]. $\operatorname{Var}\left\{\Delta x\left(t_{n}, \Delta t\right)\right\}=\propto|\Delta t|^{2 H}$, where 
$\Delta x\left(t_{n}, \Delta t\right)=x\left(t_{n}+\Delta t\right)-x\left(t_{n}\right)$ and $H$ is the Hurst exponent computed from a log-log plot using

$$
H=\lim _{\Delta t \rightarrow 0} \frac{1}{2} \frac{\log \operatorname{Var}\left\{\Delta x\left(t_{n}, \Delta t\right)\right\}}{\log (\Delta t)}
$$

Finally, the variance dimension is defined as $D_{\sigma}=E+1-H$, where $E$ is the Euclidean dimension which is one for the time series.

\subsection{Approximate entropy}

Entropy describes behavior of a system in terms of randomness, and quantifies information about underlying dynamics. The approximate Entropy (ApEn) is able to distinguish lowdimensional deterministic system, chaotic system, stochastic and mixed systems [50]. It has its roots in the work of Grassberger and Procaccia [51] and Eckmann and Ruelle [52]. A time series $x_{n}$ of length $N$ is divided into a set of $m$-length vectors $u_{m}(i)$. Then the number of vectors $u_{m}(i)$ and $u_{m}(j)$, close to each other, in an Euclidean sense $d\left[u_{m}(i), u_{m}(j)\right] \leq r$, is expressed by the number $n_{i}^{m}(r)$. This number is used to calculate the probability of vectors being close according to $C_{i}^{m}(r)=n_{i}^{n} /(N-m+1)$. Let's define the function $\Phi^{m}(r)=$ $1 /(N-m+1) \sum_{i=1}^{N-m+1} \ln C_{i}^{m}(r)$. Consequently the ApEn can be defined as

$$
\operatorname{ApEn}(m, r)=\lim _{N \rightarrow \infty}\left[\Phi^{m}(r)-\Phi^{m+1}(r)\right]
$$

Generally, the tolerance $r$ is $r=(0.15 ; 0.2) \cdot S D(S D$ stands for standard deviation) and the embedding dimension $m=2$ [22]. ApEn is broadly applicable for a data series of length $N>100$ [50]. Nevertheless, this was suggested for a wide spectrum of applications. In our case, a meaningful data length for ApEn is $N \geq 1000$.

\subsection{Sample entropy}

A slightly modified estimation of approximate entropy was proposed by [53] and resulted in sample entropy (SampEn). This estimation overcame the shortcomings of the ApEn mainly because the self-matches are excluded. Secondly, conditional probabilities are not estimated by a template-wise approach. SampEn requires only that one template finds a match of length $m+1$. The calculation of SampEn is as follows:

$$
\operatorname{SampEn}(m, r)=\lim _{N \rightarrow \infty}-\ln \frac{C^{m+1}(r)}{C^{m}(r)} .
$$

The values for parameters $r$ and $m$ were the same as for the ApEn. Also the data requirements hold.

\subsection{Lempel Ziv complexity}

The Lempel Ziv Complexity (LZC) [54] estimates reoccurring patterns contained in the time series irrespective of time. A periodic signal has the same reoccurring patterns and low complexity while in random signal individual patterns are rarely repeated and signal complexity is high.

A signal, $x(1), x(2), \ldots, x(n)$, is encoded to form a sequence $S$ such that an increase in value, $x(i+1)>x(i)$, is encoded by
1 and decrease, $x(i+1) \leq x(i)$, by 0 . The algorithm counts distinct patterns in $S$; for each new pattern the complexity $c(n)$ is increased by 1 . By convention, when the last element of $S$ is reached, the $c(n)$ is also increased by 1 . The $c(n)$ is dependent on the length of the original sequence $n$. We used the normalization form to avoid this dependence on the number of data points [54]. The normalized $C(n)$ is defined as $C(n)=c(n) \cdot \log _{2}(n) / n$. Binary encoding was used in order to avoid a dependence of results on quantification criteria and normalization procedures. The required data length for binary encoded data is 1000 samples [55].

\section{Surrogate data test}

Since the FHR is a signal with finite length, finite precision, and, more importantly, is contaminated with noise, we performed a surrogate data test. The surrogate data has the same distribution and autocorrelation function as the original data but the nonlinear properties are destroyed. We used iterative Amplitude Adjusted Fourier Transform method [56] with endpoint matching to generate 39 surrogates and tested the null hypothesis that the data originates from Gaussian linear stochastic process. All methods were used as a discriminator between original data and its surrogates; the level of significance was set to $p<0.05$.

\section{Feature selection/ dimensionality reduction and classifi- cation}

\subsection{Feature selection/ dimensionality reduction}

Since we acquired 33 features in total (11 FIGO-like, 14 HRV-based, and 8 Nonlinear features) an automatic selection of attributes was performed. First, features were separated based on their "origin" - thus we created four sets: FIGOlike (morphological features based on FIGO-guidelines), HRVbased (features inspired by adult HRV analysis features), Nonlinear (features described in Section 5), and features selected from all domains (FS-Complete). Then, based on our experience, we employed three completely unrelated feature selection/ dimensionality reduction algorithms to produce a more informative input to the classifier. Therefore we employed: Principal component analysis (PCA) [57]; Information gain (InfoGain) [58] - both implemented in WEKA [58] - and Group of adaptive models evolution neural network (GAME-NN) developed at the Czech Technical University in Prague [59].

The last step of the feature selection consisted of feature meta-selection, where only features selected by at least two methods were included in the final sets. The purpose of features division based on their "origin" was to prove that within each group there are features with information value and, hence, their computations were performed correctly.

\subsection{Feature classification and performance evaluation}

The different features sets were used to train the following classifiers: Naive Bayes, Support Vector Machine (SVM), and 

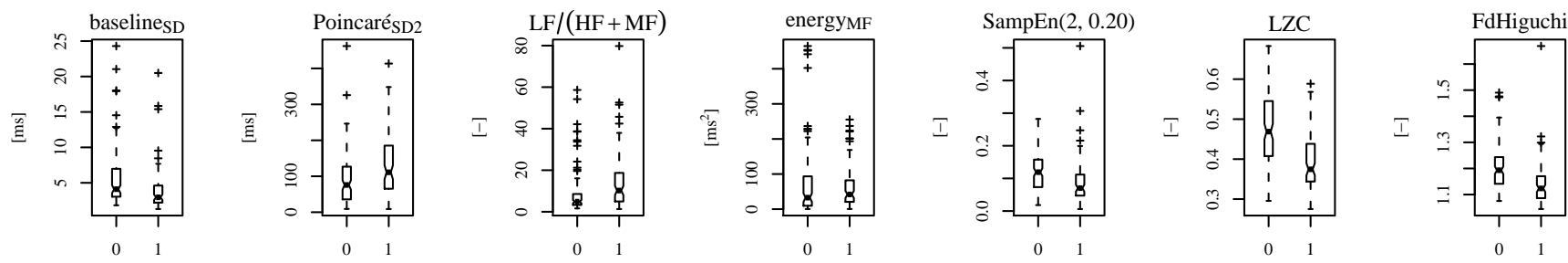

Figure 4: Distributions of selected features; 0-normal class, 1-pathological class.

C4.5 decision tree; all implemented in WEKA [58]. The description of these methods could be found e.g in [60]. Using 10 -fold cross validation the best parameters for the SVM (radial basis function kernel) were sought: the radius $\gamma=1 / 2 \sigma^{2}$ and cost parameter $\mathrm{C}$.

The most common measures for the evaluation of experimental results are: sensitivity, specificity, and F-measure. The F-measure is computed using precision (= positive predictive value) $P R=T P /(T P+F P)$ and recall (= sensitivity) $R E=$ $T P /(T P+F N)$ :

$$
F_{\beta}=\frac{\left(1+\beta^{2}\right)(P R \cdot R E)}{\beta^{2} \cdot P R+R E} .
$$

Parameters TP, FP, and FN stand for true positive, false positive and false negative, respectively. The parameter $\beta$ is usually set to one. This means that precision and recall have equal priority. Another useful measure is receiver operator curve (ROC) and the area under this curve (AUC). The ROC defines the relationship between sensitivity and specificity for the model parameters. Given the ROC and AUC we can estimate which parameters are the best for modeling and if the model is suitable for the task in the first place. The experimental results were computed using 10-fold cross validation.

The difference between individual classifiers trained using different feature sets should be statistically confirmed. Although there is no unified framework the use of McNemar's test is recommended [61]. However, when dealing with a relatively small dataset (217 records in our case) there is not enough data to acceptably minimize both errors: (i) when estimating classification performance, (ii) in statistical testing. Apparently, the better way is to minimize the former error and refrain from statistical testing.

\section{Results}

The FHR signals were analyzed by conventional and nonlinear methods. For the correlation dimension, $D_{2}$, we found optimal time delay to be equal to $\tau=3.9 \pm 2.5 \mathrm{~s}$. We performed the surrogate data test with all methods as discriminators and rejected the null hypotheses that data originate from Gaussian linear stochastic process on $p<0.05$.

We performed feature selection for each group of features with the results presented in Table 1. The distributions of features from the FS-Complete set are present in Figure 4. The discrimination between normal and pathological examples is most apparent for features: Poincaré_SD2, $\operatorname{SampEn}(2,0.20)$, and LZC. The projection of the 7-dimensional feature space (FS-Complete set features) into 2-dimensional space was performed by a self organizing map $[62,63](20 \times 20$ units in hexagonal topology and Gaussian neighborhood trained for 200 iterations of rough training phase and 200 iterations of fine tuning phase; a sequential training algorithm was used). This map is shown in Figure 5; the discrimination between pathological (red) and normal (green) examples is clearly visible, however there are also a number of cases that could not be easily separated. This corresponds to the classification accuracy shown in Table 3 below.

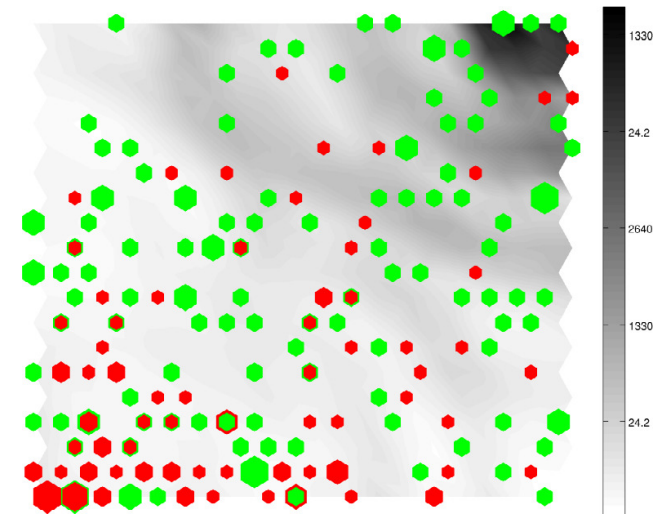

Figure 5: Projection of 7-dimensional FS-Complete feature set using self organizing map into 2-dimensions. green - normal class, red - pathological class, grey scale represents distance between examples.

Table 1: Selected features organized by their origin: FIGO-like, HRV-based; Nonlinear, FS-Complete.

\begin{tabular}{ll}
\hline Feature set & Selected features \\
\hline FIGO-like & baselineSD, meanII \\
HRV-based & $\begin{array}{l}\text { Poincaré_SD2, LF/(HF + MF), energyLF, ener- } \\
\text { gyMF, energyVLF }\end{array}$ \\
Nonlinear & SampEn(2,0.20), LZC, FdVariance, FdHiguchi \\
FS-Complete & baselineSD, Poincaré_SD2, LF/(HF + MF), en- \\
& ergyMF, SampEn(2,0.20), LZC, FdHiguchi \\
\hline
\end{tabular}

We found optimal settings for SVM (radial basis function kernel): $\gamma=0.01$, cost parameter $\mathrm{C}=14$. Results for all features and with nonlinear features excluded are presented in $\mathrm{Ta}-$ ble 2. Classification results for each feature set, described in 
Table 1, are shown in Table 3. Results based on the expert's annotations for the same data are presented in Table 4 for comparison.

Table 2: Classification results for all features and with nonlinear features excluded.

\begin{tabular}{llccc}
\hline Feature set & All in [\%] & NaiveBayes & SVM & C4.5 Tree \\
\hline \multirow{4}{*}{ All features } & Sensitivity & 67.0 & 66.0 & 55.3 \\
& Specificity & 75.6 & 78.9 & 71.5 \\
& Precision & 67.7 & 70.5 & 59.8 \\
& F-measure & 67.4 & 68.0 & 57.5 \\
& AUC & 0.74 & 0.74 & 0.63 \\
\hline \multirow{3}{*}{ Nonlinear } & Sensitivity & 60.6 & 53.2 & 54.3 \\
features & Specificity & 71.5 & 78.9 & 69.9 \\
excluded & Precision & 62.0 & 65.8 & 58.0 \\
& F-measure & 61.3 & 58.7 & 56.0 \\
& AUC & 0.69 & 0.74 & 0.64 \\
\hline
\end{tabular}

Table 3: Classification results for selected features from different groups: FIGO-like, HRV-based; Nonlinear, FS-Complete.

\begin{tabular}{llccc}
\hline Feature set & All in [\%] & NaiveBayes & SVM & C4.5 Tree \\
\hline \multirow{5}{*}{ FIGO-like } & Sensitivity & 66.0 & 53.2 & 55.3 \\
& Specificity & 68.3 & 81.3 & 65.9 \\
& Precision & 61.4 & 68.5 & 55.3 \\
& F-measure & 63.6 & 64.2 & 55.3 \\
& AUC & 0.70 & 0.71 & 0.60 \\
\hline & Sensitivity & 44.7 & 56.4 & 59.6 \\
& Specificity & 82.1 & 83.6 & 69.9 \\
& Precision & 65.6 & 72.5 & 60.2 \\
& F-measure & 53.2 & 67.4 & 59.9 \\
& AUC & 0.71 & 0.73 & 0.60 \\
\hline \multirow{5}{*}{ Nonlinear } & Sensitivity & 68.9 & 70.1 & 53.3 \\
& Specificity & 73.2 & 78.1 & 85.0 \\
& Precision & 64.6 & 71.0 & 71.6 \\
& F-measure & 66.7 & 70.6 & 61.1 \\
& AUC & 0.74 & 0.75 & 0.68 \\
\hline \multirow{6}{*}{ Complete } & Sensitivity & 72.3 & $\mathbf{7 3 . 4}$ & 60.6 \\
& Specificity & 75.6 & $\mathbf{7 6 . 3}$ & 71.5 \\
& Precision & 69.4 & $\mathbf{7 0 . 3}$ & 62.0 \\
& F-measure & 70.8 & $\mathbf{7 1 . 9}$ & 61.3 \\
& AUC & 0.75 & $\mathbf{0 . 7 8}$ & 0.68 \\
\hline \multirow{6}{*}{ Hom } & & &
\end{tabular}

When the nonlinear features were excluded and only conventional features were used the classification performance decreased as is shown in Table 2. The best results were achieved using the SVM with FS-Complete feature set, shown in bold in Table 3.

\section{Discussion}

When analyzing the FHR one has to be aware of its properties especially when using nonlinear methods. As an example we can use correlation dimension $D_{2}$. The values of $D_{2}$ for all records $D_{2}=2.42 \pm 0.61$ were low in comparison to $D_{2} \approx 6$ of adult HRV [64]. There are two possible explanations. First, the RR intervals from external (ultrasound) records were corrupted
Table 4: Classification results of clinicians when comparing their evaluation of FHR to objective $\mathrm{pH}$ annotation.

\begin{tabular}{lccc}
\hline All in [\%] & Expert \#1 & Expert \#2 & Expert \#3 \\
\hline Sensitivity pH & 34.4 & 49.0 & 40.5 \\
Specificity pH & 14.1 & 16.2 & 8.6 \\
Intra-observer agreement & 70.7 & 56.1 & 76.7 \\
\hline Inter-observer agreement & & 80.5 & \\
between the three experts & & & \\
\hline
\end{tabular}

by the measurement process, such as quantization, filtering, and averaging. To be more specific, the external monitoring uses Doppler ultrasound for fetal heart beat detection which are usually determined from a periodicity of Doppler envelope using autocorrelation function. This function tends to average slight successive changes of heart beats resulting in loss of FHR variability [65]. Second, during the preprocessing stage, artefacts were linearly interpolated, i.e. a possible complex behavior was replaced by a line. Both factors may contribute to decreased value of $D_{2}$. Nevertheless, the nonlinear methods are still applicable since the surrogate date test showed significant difference between FHR and its surrogates on $p<0.05$. However, there is also a limitation to the surrogate test due to nonlinearity introduced by the cubic spline interpolation.

Regarding the classification results there are two important questions that need to be carefully considered. How to interpret the results acquired and presented in Tables 2, 3, and 4? How to relate them to the works already done? The answer is not straightforward since there are two quite distinct approaches to processing and classification of the FHR signal intrapartum.

The first one is a more technical approach that uses the objective evaluation of the data. This approach was used in many papers during the last decade and was also adopted in this paper. Our results of $73.4 \%$ sensitivity, $76.3 \%$ of specificity, and F-measure $71.9 \%$ compare favourably to those of $[26,66]$ especially considering the small sample size they used. However, this technical approach suffers from, at least, two major drawbacks. The relation of hypoxia to the fetal cord arterial $\mathrm{pH}$ after delivery is widely discussed in several papers [31]. The predominant conclusion is that only an overall examination of the baby at about four years of age can bring a confident enough conclusion on the occurrence of effective asphyxia during the delivery. In addition, in many cases where timely interventions based on suspect/pathological FHR signal is made, the arterial $\mathrm{pH}$ values of the instrumentally delivered baby will be above the pathological threshold.

A second approach to the evaluation of FHR recordings is to acquire expert evaluation of signals and use the classification process to try to adopt an expert behavior. Nevertheless, this approach has several drawbacks as well. First, the inter and even the intra observer variabilities are quite substantial as presented in Table 4. Second, the experts categorize the signals usually according to FIGO-based guidelines into the three classes (normal, suspect, and pathological). Large subset of signals are evaluated as suspect, but suspect class does not exist after delivery, there is usually normal or (possibly) asphyxiated 
baby (about whom, there will likely not be any decisive proof for at least the next several months). Moreover, in the clinical practice there is always additional information involved in the decision making process - pushing the decision process more in one direction.

\section{Conclusion}

This paper, for the first time, evaluates the behavior of the full set of nonlinear methods on one reasonably large database. In addition we considered the usability of these methods with respect to FHR properties. We compared a full spectrum of nonlinear features to the conventional features already used in other papers, and we showed that classification based on feature sets including nonlinear features performs the best. It also performs on-par with the best results obtained in other works such as $[26,66]$.

The evaluation of FHR still remains subjective [67]. Clinicians say that they use conventional features like accelerations, decelerations, and variability though there is a contributing factor of pattern-like memory acquired during working experience. In contrast to the previous works, we proved that nonlinear features are useful in combination with conventional ones on a large database.

To be able to affect the way the data is processed in the clinical settings there is a need to include additional information describing the environment in which the FHR signal is acquired (maternal preexisting condition, drugs used, length of delivery etc.) which are an integral part of the obstetrician's decision making progress. Nevertheless, it is evident that proper processing of the FHR will remain an important part of any future decision support system.

\section{Acknowledgements}

This work was supported by the research programs No. NT11124-6/2010 of the Ministry of Health Care and by the research program MSM 6840770012 Transdisciplinary Research in the Area of Biomedical Engineering II of the CTU in Prague, sponsored by the Ministry of Education, Youth and Sports of Czech Republic.

\section{References}

[1] V. Doria, A. T. Papageorghiou, A. Gustafsson, A. Ugwumadu, K. Farrer, S. Arulkumaran, Review of the first 1502 cases of ECG-ST waveform analysis during labour in a teaching hospital, BJOG 114 (2007) 12021207.

[2] M. Westerhuis, A. Kwee, A. A. van Ginkel, A. P. Drogtrop, W. J. A. Gyselaers, G. H. A. Visser, Limitations of ST analysis in clinical practice: three cases of intrapartum metabolic acidosis, BJOG 114 (2007) 11941201 .

[3] Z. Alfirevic, D. Devane, G. M. L. Gyte, Continuous cardiotocography (CTG) as a form of electronic fetal monitoring (EFM) for fetal assessment during labour, Cochrane Database Syst Rev 3 (2006) CD006066.

[4] P. J. Steer, Has electronic fetal heart rate monitoring made a difference, Semin Fetal Neonatal Med 13 (2008) 2-7.

[5] FIGO, Guidelines for the Use of Fetal Monitoring, International Journal of Gynecology \& Obstetrics 25 (1986) 159-167.
[6] NIH, Electronic fetal heart rate monitoring: research guidelines for interpretation. National Institute of Child Health and Human Development Research Planning Workshop, Am J Obstet Gynecol 177 (1997) 13851390.

[7] J. Bernardes, A. Costa-Pereira, D. A. de Campos, H. P. van Geijn, L. Pereira-Leite, Evaluation of interobserver agreement of cardiotocograms, Int J Gynaecol Obstet 57 (1997) 33-37.

[8] O. Palomäki, T. Luukkaala, R. Luoto, R. Tuimala, Intrapartum cardiotocography - the dilemma of interpretational variation, J Perinat Med 34 (2006) 298-302.

[9] H. Norén, I. Amer-Wåhlin, H. Hagberg, A. Herbst, I. Kjellmer, K. Maršál, P. Olofsson, K. G. Rosén, Fetal electrocardiography in labor and neonatal outcome: data from the Swedish randomized controlled trial on intrapartum fetal monitoring, Am J Obstet Gynecol 188 (2003) 183-192.

[10] I. Amer-Wåhlin, K. Maršál, ST analysis of fetal electrocardiography in labor, Seminars in Fetal and Neonatal Medicine 16 (2011) 29-35.

[11] H. Gonçalves, A. P. Rocha, D. A. de Campos, J. Bernardes, Linear and nonlinear fetal heart rate analysis of normal and acidemic fetuses in the minutes preceding delivery, Med Biol Eng Comput 44 (2006) 847-855.

[12] G. Magenes, M. G. Signorini, D. Arduini, Classification of cardiotocographic records by neural networks, in: Proc. IEEE-INNS-ENNS International Joint Conference on Neural Networks IJCNN 2000, volume 3, pp. 637-641.

[13] J. Van Laar, M. Porath, C. Peters, S. Oei, Spectral analysis of fetal heart rate variability for fetal surveillance: Review of the literature, Acta Obstetricia et Gynecologica Scandinavica 87 (2008) 300-306.

[14] E. Salamalekis, P. Thomopoulos, D. Giannaris, I. Salloum, G. Vasios, A. Prentza, D. Koutsouris, Computerised intrapartum diagnosis of fetal hypoxia based on fetal heart rate monitoring and fetal pulse oximetry recordings utilising wavelet analysis and neural networks, BJOG 109 (2002) 1137-1142.

[15] G. Georgoulas, C. D. Stylios, P. P. Groumpos, Feature Extraction and Classification of Fetal Heart Rate Using Wavelet Analysis and Support Vector Machines, International Journal on Artificial Intelligence Tools 15 (2005) 411-432.

[16] P. Warrick, E. Hamilton, D. Precup, R. Kearney, Classification of normal and hypoxic fetuses from systems modeling of intrapartum cardiotocography, IEEE Transactions on Biomedical Engineering 57 (2010) 771-779.

[17] D. G. Chaffin, C. C. Goldberg, K. L. Reed, The dimension of chaos in the fetal heart rate, Am J Obstet Gynecol 165 (1991) 1425-1429.

[18] N. A. Gough, Fractal analysis of foetal heart rate variability, Physiol Meas 14 (1993) 309-315.

[19] C. S. Felgueiras, J. P. de Sá, J. Bernardes, S. Gama, Classification of foetal heart rate sequences based on fractal features, Med Biol Eng Comput 36 (1998) 197-201.

[20] A. Kikuchi, N. Unno, T. Horikoshi, T. Shimizu, S. Kozuma, Y. Taketani, Changes in fractal features of fetal heart rate during pregnancy, Early Hum Dev 81 (2005) 655-661.

[21] P. Hopkins, N. Outram, N. Lofgren, E. C. Ifeachor, K. G. Rosen, A Comparative Study of Fetal Heart Rate Variability Analysis Techniques, in: Proc. 28th Annual International Conference of the IEEE Engineering in Medicine and Biology Society EMBS '06, pp. 1784-1787.

[22] S. M. Pincus, R. R. Viscarello, Approximate entropy: a regularity measure for fetal heart rate analysis, Obstet Gynecol 79 (1992) 249-255.

[23] M. Ferrario, M. G. Signorini, G. Magenes, S. Cerutti, Comparison of entropy-based regularity estimators: application to the fetal heart rate signal for the identification of fetal distress, IEEE Trans Biomed Eng 53 (2006) 119-125.

[24] H. Gonçalves, J. Bernardes, A. P. Rocha, D. A. de Campos, Linear and nonlinear analysis of heart rate patterns associated with fetal behavioral states in the antepartum period, Early Hum Dev 83 (2007) 585-591.

[25] M. Ferrario, M. Signorini, G. Magenes, Complexity analysis of the fetal heart rate variability: Early identification of severe intrauterine growthrestricted fetuses, Medical and Biological Engineering and Computing 47 (2009) 911-919.

[26] G. Georgoulas, C. D. Stylios, P. P. Groumpos, Predicting the risk of metabolic acidosis for newborns based on fetal heart rate signal classification using support vector machines, IEEE Trans Biomed Eng 53 (2006) 875-884.

[27] G. Georgoulas, D. Gavrilis, I. G. Tsoulos, C. D. Stylios, J. Bernardes, P. P. Groumpos, Novel approach for fetal heart rate classification intro- 
ducing grammatical evolution, Biomedical Signal Processing and Control 2 (2007) 69-79.

[28] D. A. de Campos, P. Sousa, A. Costa, J. Bernardes, Omniview-SisPorto 3.5 - A central fetal monitoring station with online alerts based on computerized cardiotocogram $+\mathrm{ST}$ event analysis, Journal of Perinatal Medicine 36 (2008) 260-264.

[29] B. Guijarro-Berdinas, A. Alonso-Betanzos, Empirical evaluation of a hybrid intelligent monitoring system using different measures of effectiveness, Artif Intell Med 24 (2002) 71-96.

[30] A. Sundström, D. Rosén, K. Rosén, Fetal Surveillance - textbook, [online] Gothenburg, Sweden: Neoventa Medical AB, 2000.

[31] H. Cao, D. E. Lake, I. Ferguson, J. E., C. A. Chisholm, M. P. Griffin, J. R. Moorman, Toward quantitative fetal heart rate monitoring, IEEE Trans Biomed Eng 53 (2006) 111-118.

[32] G. Georgoulas, C. Stylios, G. Nokas, P. Groumpos, Classification of fetal heart rate during labour using hidden Markov models, in: Neural Networks, 2004. Proceedings. 2004 IEEE International Joint Conference on, volume 3, pp. $2471-2475$.

[33] J. Bernardes, C. Moura, J. P. de Sa, L. P. Leite, The Porto system for automated cardiotocographic signal analysis, J Perinat Med 19 (1991) 61-65.

[34] D. K. Kahaner, C. Moler, S. Nash, Numerical Methods and Software, Prentice-Hall, 1989.

[35] J. C. Sprott, Chaos and Time-Series Analysis, Oxford University Press, 2003.

[36] M. Cesarelli, M. Romano, M. Ruffo, P. Bifulco, G. Pasquariello, A. Fratini, PSD modifications of FHRV due to interpolation and CTG storage rate, Biomedical Signal Processing and Control In Press, Corrected Proof (2010) -.

[37] M. G. Signorini, G. Magenes, S. Cerutti, D. Arduini, Linear and nonlinear parameters for the analysis of fetal heart rate signal from cardiotocographic recordings, IEEE Trans Biomed Eng 50 (2003) 365-374.

[38] J. Bernardes, H. Gonçalves, D. Ayres-De-Campos, A. Rocha, Sex differences in linear and complex fetal heart rate dynamics of normal and acidemic fetuses in the minutes preceding delivery, Journal of Perinatal Medicine 37 (2009) 168-176.

[39] Task-Force, Heart rate variability. Standards of measurement, physiological interpretation, and clinical use. Task Force of the European Society of Cardiology and the North American Society of Pacing and Electrophysiology, Eur Heart J 17 (1996) 354-381.

[40] A. Goldberger, V. Bhargava, B. West, A. Mandell, On the mechanism of cardiac electrical stability. The fractal hypothesis, Biophysical Journal 48 (1985) 525-528.

[41] L. Glass, Introduction to controversial topics in nonlinear science: is the normal heart rate chaotic?, Chaos 19 (2009) 028501.

[42] F. Takens, Detecting strange attractors in turbulence, Dynamical Systems and Turbulence 4 (1981) 366-381.

[43] A. M. Fraser, H. L. Swinney, Independent coordinates for strange attractors from mutual information, Physical Review A 33(2) (1986) 11341140

[44] L. Cao, Practical method for determining the minimum embedding dimension of a scalar time series, Physica D 110 (1997) 43-50.

[45] D. Ruelle, Deterministic Chaos: The Science and the Fiction, Proc. R. Soc. London 427 (1990) 241-248.

[46] F. Takens, On the Numerical Determination of the Dimension of an Attractor, Dynamical Systems and Bifurcations 1125 (1985) 99-106.

[47] J. Theiler, Lacunarity in a best estimator of fractal dimension, Physics Letters A 133 (1988) 195-200.

[48] T. Higuchi, Approach to an irregular time series on the basis of the fractal theory, Phys. D 31 (1988) 277-283.

[49] W. Kinsner, Batch and real-time computation of a fractal dimension based on variance of a time series., Technical Report, Department of Electrical \& Computer Engineering, University of Manitoba, Winnipeg, Canada, 1994.

[50] S. Pincus, Approximate entropy (ApEn) as a complexity measure, Chaos 5 (1) (1995) 110-117.

[51] P. Grassberger, I. Procaccia, Measuring the strangeness of strange attractors, Physica D: Nonlinear Phenomena 9 (1983) 189-208.

[52] J. P. Eckmann, D. Ruelle, Ergodic theory of chaos and strange attractors, Rev. Mod. Phys. 57 (1985) 617-656

[53] J. S. Richman, J. R. Moorman, Physiological time-series analysis using approximate entropy and sample entropy, Am J Physiol Heart Circ Physiol 278 (2000) H2039-H2049.

[54] A. Lempel, J. Ziv, On the complexity of finite sequences, IEEE Transactions on Information Theory IT-22 (1) (1976) 75-81.

[55] M. Ferrario, M. G. Signorini, S. Cerutti, Complexity analysis of 24 hours heart rate variability time series, Conf Proc IEEE Eng Med Biol Soc 6 (2004) 3956-3959.

[56] T. Schreiber, A. Schmitz, Improved Surrogate Data for Nonlinearity Tests, Phys. Rev. Lett. 77 (4) (1996) 635-638.

[57] C. Bishop, Neural networks for pattern recognition, Clarendon Press, Oxford, 1995.

[58] I. H. Witten, E. Frank, Data Mining: Practical machine learning tools and techniques, Morgan Kaufmann, San Francisco, 2005.

[59] P. Kordik, Fully Automated Knowledge Extraction using Group of Adaptive Models Evolution, Ph.D. thesis, Czech Technical University in Prague, FEE, Dep. of Comp. Sci. and Computers, FEE, CTU Prague, Czech Republic, 2006.

[60] R. O. Duda, P. E. Hart, D. G. Stork, Pattern Classification (2nd Edition), Wiley-Interscience, 2000.

[61] S. Salzberg, On Comparing Classifiers: Pitfalls to Avoid and a Recommended Approach, Data Min. Knowl. Discov. 1 (1997) 317-328.

[62] T. Kohonen, Self-Organizing Maps, volume 30, Series in Information Sciences, Springer, Heidelberg, 2001.

[63] J. Vesanto, J. Himberg, E. Alhoniemi, J. Parhankangas, SOM Toolbox for Matlab 5, Helsinki University of Technology, Report A57, Libella Oy Espoo (2000).

[64] P. Van Leeuwen, H. Bettermann, U. An der Heiden, H. Kummell, Circadian aspects of apparent correlation dimension in human heart rate dynamics, American Journal of Physiology - Heart and Circulatory Physiology 269 (1995) 130-134.

[65] J. Jezewski, J. Wrobel, K. Horoba, Comparison of Doppler ultrasound and direct electrocardiography acquisition techniques for quantification of fetal heart rate variability, IEEE Trans Biomed Eng 53 (2006) 855864

[66] T. K. Chung, M. P. Mohajer, Z. J. Yang, A. M. Chang, D. S. Sahota, The prediction of fetal acidosis at birth by computerised analysis of intrapartum cardiotocography, Br J Obstet Gynaecol 102 (1995) 454-460.

[67] I. Amer-Wåhlin, K. Källén, A. Herbst, H. Rydhstroem, A. Sundström, K. Maršál, Implementation of new medical techniques: experience from the Swedish randomized controlled trial on fetal ECG during labor, J Matern Fetal Neonatal Med 18 (2005) 93-100. 